\title{
Strong evidence for the three-dimensional Fermi liquid behaviour of quasiparticles in high- $T_{\mathrm{C}}$ cupurates
}

\author{
Setsuo Misawa ${ }^{1}$ \\ Institute of Quantum Science, Nihon University, Kanda-Surugadai, Tokyo 101-8308, Japan
}

\begin{abstract}
It is generally believed that behaviours of quasiparticles (holes) in high- $T_{\mathrm{C}}$ cupurates should be attributed to the two-dimensional(2D) electronic states in the $\mathrm{CuO}_{2}$ planes. The various anomalies of the transport coefficients for temperatures above $T_{\mathrm{C}}$ are long-standing insoluble puzzles and cause serious controversy. Here we reanalyse the published experimental date of LSCO cupurates. We find that the normal-state susceptibility, resistivity, Hall coefficient etc vary precisely as $T^{2} \ln T$ as a function of temperature $T$ in agreement with the prediction of the Fermi liquid model. The quasiparticles are shown to definitely behave as a 3D Fermi liquid. Various attempts to describe the system in terms of non-Fermi liquids,e.g. the RVB state, seem to be erroneous.
\end{abstract}

Key words: High- $T_{\mathrm{C}}$ cupurate; Normal state; Fermi liquid; Three-dimension; Resistivity

High- $T_{\mathrm{C}}$ cupurates are characterized by their anomalous properties in the normal state. The normal state resistivity often varies almost linearly with temperature $T$. To clarify the true nature of the normal state is a clue to understanding the superconductivity of this system. Many authors claim that the anomalies should be attributed to non-Fermi liquids. In contrast to this, here we present strong and conclusive evidence to show that quasiparticles (holes) of the system form a three-dimensional(3D) Fermi liquid, and hence the anomalous properties can be precisely described by the Fermi liquid model proposed by the present author in 1970 [1]. Although the system is highly anisotropic, as will be seen below, the $3 \mathrm{D}$ nature of the quasiparticles is of critical importance. We have already shown that this model explains universally the physics of paramagnetic d-metals, $\mathrm{MnSi}(\mathrm{FeSi})$, Laves phase compounds and heavy fermion compounds.

Because of the presence of the Fermi surface and of the interactions, 3D Fermi liquids produce various logarithmic (nonanalytic) terms; the quasiparticle energy

\footnotetext{
1 Corresponding author. Tel and Fax: +81-1-3259-0648

E-mail: misawa@phys.cst.nihon-u.ac.jp
}

contains, as a function of momentum $p$ with the Fermi momentum $p_{0}$, a $\left(p-p_{0}\right)^{3} \ln \left|p-p_{0}\right|$ term which creates a $\varepsilon^{2} \ln |\varepsilon|$ term in the density of states function, where $\varepsilon$ is the energy measured from the Fermi surface. Thus the electrical conductivity due to impurities and/or imperfections is given by $\sigma_{\mathrm{imp}}=\sigma_{0}+\sigma_{1} T^{2} \ln \left(T / T_{1}\right)$. The total resistivity $\rho$ is a sum of the proper $\gamma T^{2}$ resistivity arising from the Umklapp Coulomb scattering and the impurity resistivity $\rho_{\mathrm{imp}}=\sigma_{\mathrm{imp}}{ }^{-1} ; \rho(T)=$ $\rho_{0}-\rho_{1} T^{2} \ln \left(T / T_{\rho}^{*}\right)$, where $\rho_{0}=\sigma_{0}{ }^{-1}, \rho_{1}=\sigma_{1} / \sigma_{0}{ }^{2}$ and $T_{\rho}^{*}=T_{1} \exp \left(\sigma_{0}{ }^{2} \gamma / \sigma_{1}\right)$. In contrast to the general belief, $\rho$ varies as $T^{2} \ln \left(T / T_{\rho}^{*}\right)$, where temperature $T_{\rho}^{*}$ (or $T_{1}$ ) has no particular physical meaning and takes any value, for it arises from a sum of $T^{2}$ and $T^{2} \ln T$ terms. Here it is important to note that $T^{2} \ln \left(T / T_{\rho}^{*}\right)$ varies almost linearly with $T$ for $0.1 \lesssim T / T_{\rho}^{*} \lesssim 0.4$. By the same reasoning, the Hall coefficient varies as $T^{2} \ln T$. Because of the short-wavelength density fluctuations the spin antisymmetric part of the Landau f-function contains a $\left(p-p_{0}\right)^{2} \ln \left|p-p_{0}\right|$ term which causes the $T^{2} \ln T$ variation of the magnetic susceptibility $\chi ; \chi(T)=\chi(0)-b T^{2} \ln \left(T / T^{*}\right) ; \chi(T)$ should exhibit a maximum at temperature $T_{\max }=T^{*} / \sqrt{e}$. Here again $T_{\max }$ has no particular meaning; it does not rep- 


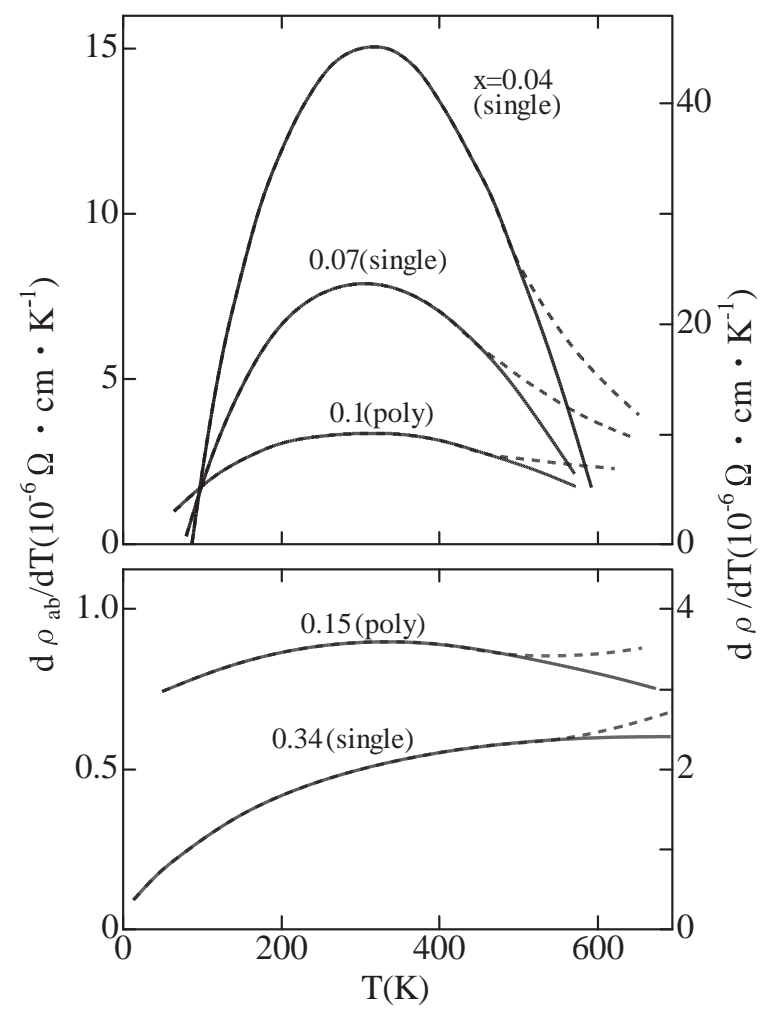

Fig. 1. $d \rho / d T$ vs $T$ of single-crystal (left scale) and polycrystalline (right scale) $\mathrm{La}_{2-x} \mathrm{Sr}_{x} \mathrm{CuO}_{4}$. The smoothed experimental date [3] fit precisely theoretical $T \ln T$ curves (solid lines) up to about $500 \mathrm{~K}$ above which the date (dashed lines) deviate from the solid lines.

resent the strength of antiferromagnetic correlations.

To confirm the above view, we reanalyse the published experimental data of $\mathrm{La}_{2-x} \mathrm{Sr}_{x} \mathrm{CuO}_{4}$ cupurates for a wide range of $x$. References [2,3] are selected on the basis of good samples and mutual consistency of the data. Figure 1 shows that the experimental data of $d \rho / d T$ follow precisely a $T \ln T$ law, and hence $\rho-\rho_{0} \sim$ $T^{2} \ln T$, up to about $500 \mathrm{~K}$ above which higher order terms are needed. In fact, as shown in Fig.2, the resistivity data can be precisely fitted by $\rho(T)=\rho_{0}-$ $\rho_{1} T^{2} \ln \left(T / T_{\rho}^{*}\right)-\rho_{2} T^{4} \ln \left(T / T_{\rho}^{* *}\right)$ up to $1000 \mathrm{~K}$. The latest experimental data [4] of the in-plane and out-ofplane resistivities for high quality single crystals also support this law. Similarly the Hall coefficient and Hall angle are shown to vary as $T^{2} \ln T$. Figure 3 shows that the variation of $\chi(T)$ follows a $T^{2} \ln T$ law; if the system were a $2 \mathrm{D}$ Fermi liquid, $\chi(T)$ would vary linearly with $T$ [5]. Surprisingly this law is valid continuously up to $x=0$; some parts of holes in $\mathrm{La}_{2} \mathrm{CuO}_{4}$ behave as a $3 \mathrm{D}$ Fermi liquid.

In conclusion, the normal state properties of high$T_{\mathrm{C}}$ cupurates can be thoroughly described within the framework of the 3D Fermi liquid. The concept of nonFermi liquids is not needed at all.

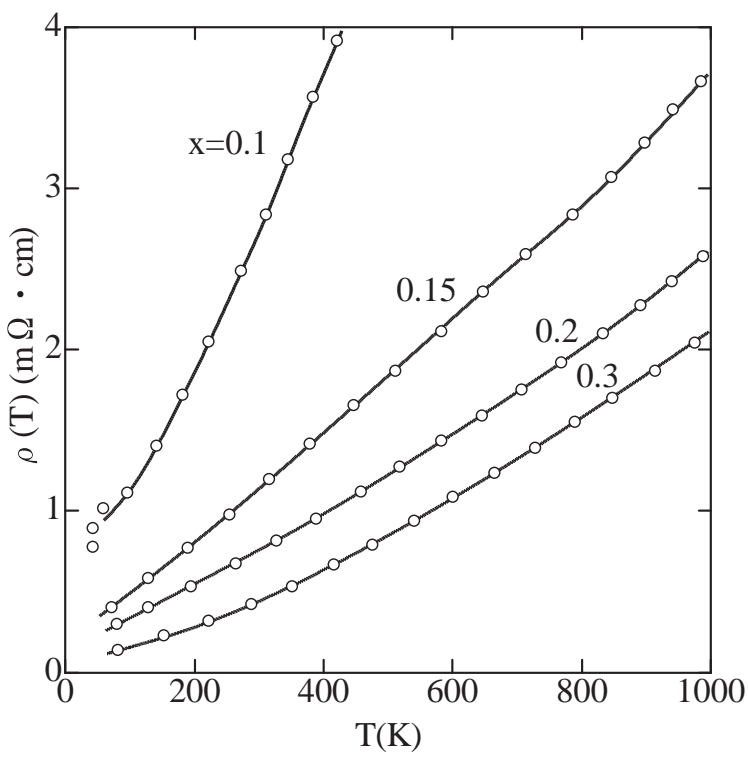

Fig. 2. $\rho(T)$ vs $T$ of polycrystalline $\mathrm{La}_{2-x} \mathrm{Sr}_{x} \mathrm{CuO}_{4}$. Open circles; representative points of the experimental date [3]: Solid lines; theoretical curves.

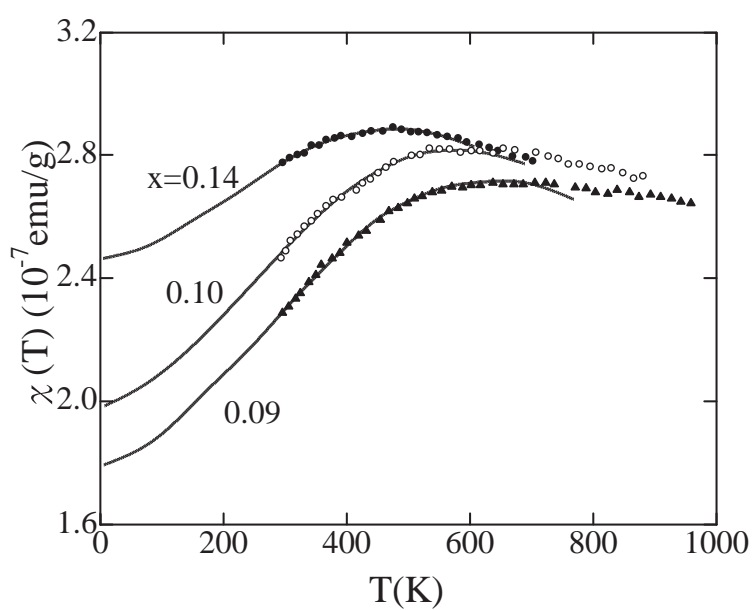

Fig. 3. $\chi(T)$ vs $T$ of $\mathrm{La}_{2}-x \mathrm{Sr}_{x} \mathrm{CuO}_{4}$. Symbols; experimental data [2]: Solid lines; theoretical curves.

References

[1] S. Misawa, Phys. Lett. A32 (1970) 153; J. Magn. Magn. Mater. 76 \& 77 (1988) 356; Europhys. Lett. 14 (1991) 377; Physica B 211 (1995) 158; J. Magn. Magn. Mater. 177-181 (1998) 325; J. Phys. Soc. Jpn. 68 (1999) 32.

[2] R. Yoshizaki et al., Physica C 166 (1990) 417.

[3] H. Takagi et al., Phys. Rev. Lett. 69 (1992) 2975; H.Y. Hwang et al., Phys. Rev. Lett. 72 (1994) 2636.

[4] S. Kojima et al., Proc. of LT23 (Physica C),2002.

[5] S. Misawa, J. Phys. Soc. Jpn. 68 (1999) 2172. 